\title{
Generalized Eulerian Numbers
}

\author{
Alfred Wünsche \\ Institut für Physik, Humboldt-Universität, Berlin, Germany \\ Email: alfred.wuensche@physik.hu-berlin.de
}

How to cite this paper: Wünsche, A. (2018) Generalized Eulerian Numbers. Advances in Pure Mathematics, 8, 335-361. https://doi.org/10.4236/apm.2018.83018

Received: February 12, 2018

Accepted: March 26, 2018

Published: March 29, 2018

Copyright $\odot 2018$ by author and Scientific Research Publishing Inc. This work is licensed under the Creative Commons Attribution International License (CC BY 4.0).

http://creativecommons.org/licenses/by/4.0/ cc) (i) Open Access

\begin{abstract}
We generalize the Eulerian numbers $\left\langle\begin{array}{l}k \\ l\end{array}\right\rangle$ to sets of numbers $E_{\mu}(k, l)$, $(\mu=0,1,2, \cdots)$ where the Eulerian numbers appear as the special case $\mu=1$. This can be used for the evaluation of generalizations $G_{\mu}(k ; z)$ of the Geometric series $G_{0}(k ; z)=G_{1}(0 ; z)$ by splitting an essential part $(1-z)^{-(\mu k+1)}$ where the numbers $E_{\mu}(k, l)$ are then the coefficients of the remainder polynomial. This can be extended for non-integer parameter $k$ to the approximative evaluation of generalized Geometric series. The recurrence relations $k \rightarrow k+1$ and $\mu \rightarrow \mu+1$ for the Generalized Eulerian numbers are derived. The Eulerian numbers $E_{1}(k, l)$ are related to the Stirling numbers of second kind $S(k, l)$ and we give proofs for the explicit relations of Eulerian to Stirling numbers of second kind in both directions. We discuss some ordering relations for differentiation and multiplication operators which play a role in our derivations and collect this in Appendices.
\end{abstract}

\section{Keywords}

Eulerian Numbers, Eulerian Polynomials, Stirling Numbers,

Permutations, Binomials, Hypergeometric Functions, Geometric Series,

Vandermonde's Convolution Identity, Recurrence Relations, Operator

Orderings

\section{Introduction}

The Eulerian numbers are discussed in the remarkable monograph of Riordan [1] from a combinatorial view and are the special topics in the recently published voluminous and versatile monograph of Petersen [2] with huge material and relations to other topics and with a great number of citations. The last contains 
also some remarks about the history of these numbers. The Eulerian numbers are taken into account in the article of Bressoud [3] in the NIST Handbook of Mathematical Functions [4]. In the interesting book of Conway and Guy [5], the "Eulerian numbers" are also shortly mentioned with one formula but without explicitly giving tables. There exist also an article about the Eulerian numbers in Weisstein's Encyclopedia of Mathematics [6].

We met the Eulerian numbers $A(k, l),(k=0,1,2, \cdots ; l=0,1,2, \cdots)$ first in quantum-mechanical calculations of some expectation values for coherent phase states and in cumulant expansions of the distance to an initial state in the time evolution of this state for the Hamiltonian of a harmonic oscillator [7]. To this time, we looked for references where these numbers which we calculated explicitly are present in literature and found them in the monograph of Riordan [1] about combinatorics ${ }^{1}$. Meanwhile, there appeared the already mentioned monograph of Petersen [2] which provided us a convenient access to this topics.

Bressoud and Petersen denote the Eulerian numbers by $\left\langle\begin{array}{l}k \\ l\end{array}\right\rangle$ similar to the binomial numbers $\left(\begin{array}{l}k \\ l\end{array}\right)$ and likely based on earlier sources. The Eulerian numbers may be ordered in form of a triangle (Eulerian triangle) in analogy to the Pascal triangle and with a similar palindromic symmetry. The notation of Petersen and of others [6] is related to the notation of Riordan [1] by $\left\langle\begin{array}{l}k \\ l\end{array}\right\rangle=A(k, l+1)$ but concerning the definition of $k$ and $l$ the first as it seems to us is preferable and we denote it by $E_{1}(k, l)=\left\langle\begin{array}{c}k \\ l\end{array}\right\rangle$ since we generalize it to numbers $E_{\mu}(k, l)$ with arbitrary $\mu \geq 0$.

The Eulerian numbers possess a combinatorial background although not introduced in this way by Euler [1] [2]. They make a subdivision of the whole number of $k$ ! permutations of $k$ elements in non-intersecting sets of permutations with the same number $l$ of descents and what counted are the Eulerian numbers $\left\langle\begin{array}{l}k \\ l\end{array}\right\rangle$. A descent in a permutation is present if from two adjacent numbers in a permutation, the first is larger than the second. This explains why the sum of Eulerian numbers of $k$ elements is equal to the total number of $k$ ! permutations and why the Eulerian numbers are symmetric with respect to descent and ascents.

As mentioned, in the present work we generalize the Eulerian numbers to sets of numbers $E_{\mu}(k, l)$ with interesting properties where the Eulerian numbers

${ }^{1}$ In the Russian translation of this monograph which we used they were translated as "Euler numbers" but since these did not be the better known numbers which are usually understood under this name we called them "Eulerian numbers" without being aware that in English they are really called in this way. 
are identical with our special case $E_{1}(k, l) \equiv\left\langle\begin{array}{l}k \\ l\end{array}\right\rangle$. Our primary intention was to obtain approximations in the evaluation of series from type of generalized Geometric series where the number $k$ in $E_{\mu}(k, l)$ is not necessarily an integer (Section 3). Such series with $k=\frac{1}{2}$ appear in quantum optics for coherent phase states in the calculation of some kind of expectation values. We discuss some properties of the numbers $E_{\mu}(k, l)$ and derive the recurrence relations for them. Furthermore, we find close relations between these numbers and the Stirling numbers of second kind. In Appendices, we discuss how some needed ordering relations for differentiation and multiplication operators are related with the Stirling numbers.

\section{Introduction of Generalized Eulerian Numbers by Evaluation of Generalized Geometric Series}

We present in this Section our concept of the introduction of Generalized Eulerian numbers $E_{\mu}(k, l)$ from the evaluation of generalized Geometric series and give explicit results and tables but without the proofs which we develop in the following Sections.

The Geometric series as special case of the Hypergeometric Functions ${ }_{1} \mathrm{~F}_{0}(a ;-; z)$ (special case of general ${ }_{p} \mathrm{~F}_{q}\left(a_{1}, \cdots, a_{p} ; c_{1}, \cdots, c_{q} ; z\right)$ )

$$
\begin{aligned}
& \sum_{n=0}^{\infty} z^{n}=\frac{1}{1-z} \equiv{ }_{1} \mathrm{~F}_{0}(1 ;-; z), \\
& \left({ }_{1} \mathrm{~F}_{0}(a ;-; z) \equiv \sum_{n=0}^{\infty} \frac{(n+a-1) !}{(a-1) !} \frac{z^{n}}{n !}=\frac{1}{(1-z)^{a}}\right) .
\end{aligned}
$$

The convergence region of this series in the complex plane is $|z|<1$.

In our concept we consider the following types of generalizations $G_{\mu}(k ; z)$ of the Geometric series with index $\mu$ and with $k$ as two parameters

$$
\begin{aligned}
& \sum_{n=0}^{\infty} 1^{k} z^{n}=\frac{1}{(1-z)^{1}} \sum_{l=0}^{0} E_{0}(k, l) z^{l} \equiv G_{0}(k ; z), \\
& \sum_{n=0}^{\infty}(n+1)^{k} z^{n}=\frac{1}{(1-z)^{k+1}} \sum_{l=0}^{k} E_{1}(k, l) z^{l} \equiv G_{1}(k ; z), \\
& \sum_{n=0}^{\infty}((n+2)(n+1))^{k} z^{n}=\frac{1}{(1-z)^{2 k+1}} \sum_{l=0}^{2 k} E_{2}(k, l) z^{l} \equiv G_{2}(k ; z), \\
& \sum_{n=0}^{\infty}((n+3)(n+2)(n+1))^{k} z^{n}=\frac{1}{(1-z)^{3 k+1}} \sum_{l=0}^{3 k} E_{3}(k, l) z^{l} \equiv G_{3}(k ; z),
\end{aligned}
$$

where $G_{0}(k ; z)=G_{1}(0 ; z)$ is the Geometric series.

The coefficients $E_{\mu}(k, l)$ in the expansion of the separated functions $E_{\mu}(k ; z)$ in powers of $z$ are given by 


$$
\begin{aligned}
& E_{0}(k, l)=\sum_{j=0}^{l} \frac{(-1)^{j} 1 !}{j !(1-j) !}=\delta_{l, 0},\left(E_{0}(k, 0)=1, E_{0}(k, l)=0 ;(l=1,2, \cdots)\right), \\
& E_{1}(k, l)=\sum_{j=0}^{l} \frac{(-1)^{j}(k+1) !}{j !(k+1-j) !}(l+1-j)^{k} \equiv\left\langle\begin{array}{c}
k \\
l
\end{array}\right\rangle, \\
& E_{2}(k, l)=\sum_{j=0}^{l} \frac{(-1)^{j}(2 k+1) !}{j !(2 k+1-j) !}((l+2-j)(l+1-j))^{k}, \\
& E_{3}(k, l)=\sum_{j=0}^{l} \frac{(-1)^{j}(3 k+1) !}{j !(3 k+1-j) !}((l+3-j)(l+2-j)(l+1-j))^{k},
\end{aligned}
$$

The numbers $\left\langle\begin{array}{l}k \\ l\end{array}\right\rangle$ here denoted by $E_{1}(k, l)$ are the Eulerian numbers. The second of the relations (2.2) for $G_{1}(k ; z)$ is called the Carlitz identity (Petersen [2], Equation (1.10 on p. 10) and the second of the relations (2.3) is the explicit sum representation of the Eulerian numbers ([2], Equation (1.11) on p. 12). The sequence of numbers $E_{\mu}(k, l)$ with $\mu=2,3, \cdots$ represents a generalization of the Eulerian numbers for integer parameter $k$ but it is possible to extend it for non-integer $k$.

The generalization of (2.2) with integer indices $\mu=0,1,2, \cdots$ is

$$
G_{\mu}(k ; z) \equiv \sum_{n=0}^{\infty}\left(\frac{(n+\mu) !}{n !}\right)^{k} z^{n} \equiv \frac{E_{\mu}(k ; z)}{(1-z)^{\mu k+1}} \equiv \frac{1}{(1-z)^{\mu k+1}} \sum_{l=0}^{\mu k} E_{\mu}(k, l) z^{l},
$$

with the polynomials $E_{\mu}(k ; z)$ of degree $\mu k$ or smaller

$$
E_{\mu}(k ; z) \equiv \sum_{l=0}^{\mu k} E_{\mu}(k, l) z^{l} \Rightarrow E_{\mu}(k ; z=1)=\sum_{l=0}^{\mu k} E_{\mu}(k, l) .
$$

Explicitly one finds for the coefficients $E_{\mu}(k, l)$ of these polynomials

$$
E_{\mu}(k, l) \equiv \sum_{j=0}^{l} \frac{(-1)^{j}(\mu k+1) !}{j !(\mu k+1-j) !}\left(\frac{(l-j+\mu) !}{(l-j) !}\right)^{k} .
$$

The introduction of the functions $E_{\mu}(k ; z)$ is connected with an evaluation of the series $G_{\mu}(k ; z)$ under separation of an essential part $(1-z)^{-(\mu k+1)}$ from remainder polynomials $E_{\mu}(k ; z)$ in $z$ which could be called Generalized Eulerian polynomials. In particular, this separation is effective for evaluations if $z$ comes near to the boundary $|z|=1$ of convergence of the series.

We give now short tables (Tables 1-4) of the numbers $E_{\mu}(k, l)$ up to $\mu=3$ which are easily to calculate by a computer:

In principle, the tables for $\mu \geq 2$ could be reduced for the common factors $\mu !^{k}$ in the $k$-th line.

We have the following relations of the Eulerian numbers $E_{1}(k, l)$ to notations in [3] [2] and in [1] [5]

$$
E_{1}(k, l) \equiv\left\langle\begin{array}{l}
k \\
l
\end{array}\right\rangle \equiv A_{k, l+1} \equiv A(k, l+1)
$$


Table 1. Numbers $E_{0}(k, l)$.

\begin{tabular}{cccccccccccc}
\hline$k$ & $l=0$ & $l=1$ & $l=2$ & $l=3$ & $l=4$ & $l=5$ & $l=6$ & $l=7$ & $l=8$ & $l=9$ & $\sum_{l=0}^{k} E_{0}(k, l)$ \\
\hline 0 & 1 & 0 & 0 & 0 & 0 & 0 & 0 & 0 & 0 & 0 & $1=0 !$ \\
1 & 1 & 0 & 0 & 0 & 0 & 0 & 0 & 0 & 0 & 0 & $1=0 !$ \\
2 & 1 & 0 & 0 & 0 & 0 & 0 & 0 & 0 & 0 & 0 & $1=0 !$ \\
$\vdots$ & $\vdots$ & $\vdots$ & $\vdots$ & $\vdots$ & $\vdots$ & $\vdots$ & $\vdots$ & $\vdots$ & $\vdots$ & $\vdots$ & $\vdots$ \\
\hline
\end{tabular}

Table 2. Numbers $E_{1}(k, l) \equiv\left\langle\begin{array}{l}k \\ l\end{array}\right\rangle$ (Eulerian numbers).

\begin{tabular}{cccccccccccc}
\hline$k$ & $l=0$ & $l=1$ & $l=2$ & $l=3$ & $l=4$ & $l=5$ & $l=6$ & $l=7$ & $l=8$ & $l=9$ & $\sum_{l=0}^{k} E_{1}(k, l)$ \\
\hline 0 & 1 & 0 & 0 & 0 & 0 & 0 & 0 & 0 & 0 & 0 & $1=0 !$ \\
1 & 1 & 0 & 0 & 0 & 0 & 0 & 0 & 0 & 0 & 0 & $1=1 !$ \\
2 & 1 & 1 & 0 & 0 & 0 & 0 & 0 & 0 & 0 & 0 & $2=2 !$ \\
3 & 1 & 4 & 1 & 0 & 0 & 0 & 0 & 0 & 0 & 0 & $6=3 !$ \\
4 & 1 & 11 & 11 & 1 & 0 & 0 & 0 & 0 & 0 & 0 & $24=4 !$ \\
5 & 1 & 26 & 66 & 26 & 1 & 0 & 0 & 0 & 0 & 0 & $120=5 !$ \\
6 & 1 & 57 & 302 & 302 & 57 & 1 & 0 & 0 & 0 & 0 & $720=6 !$ \\
7 & 1 & 120 & 1191 & 2416 & 1191 & 120 & 1 & 0 & 0 & 0 & $5040=7 !$ \\
8 & 1 & 247 & 4293 & 15619 & 15619 & 4293 & 247 & 1 & 0 & 0 & $40320=8 !$ \\
9 & 1 & 502 & 14608 & 88234 & 156190 & 88234 & 14608 & 502 & 1 & 0 & $362880=9 !$ \\
\hline
\end{tabular}

Table 3. Numbers $E_{2}(k, l)$ (common factors $2 !^{k}$ in the $k$-th lines).

\begin{tabular}{ccccccccccc}
\hline$k$ & $l=0$ & $l=1$ & $l=2$ & $l=3$ & $l=4$ & $l=5$ & $l=6$ & $l=7$ & $l=8$ & $\sum_{l=0}^{k} E_{2}(k, l)$ \\
\hline 0 & 1 & 0 & 0 & 0 & 0 & 0 & 0 & 0 & 0 & $1=0 !$ \\
1 & 2 & 0 & 0 & 0 & 0 & 0 & 0 & 0 & 0 & $2=2 !$ \\
2 & 4 & 16 & 4 & 0 & 0 & 0 & 0 & 0 & 0 & $24=4 !$ \\
3 & 8 & 160 & 384 & 160 & 8 & 0 & 0 & 0 & 0 & $720=6 !$ \\
4 & 16 & 1152 & 9648 & 18688 & 9648 & 1152 & 16 & 0 & 0 & $40320=8 !$ \\
5 & 32 & 7424 & 165056 & 885248 & 1513280 & 885248 & 165056 & 7424 & 32 & $3628800=10 !$ \\
\hline
\end{tabular}

Table 4. Numbers $E_{3}(k, l)$ (common factors $3 !^{k}$ in the $k$-th lines).

\begin{tabular}{ccccccccccc}
\hline$k$ & $l=0$ & $l=1$ & $l=2$ & $l=3$ & $l=4$ & $l=5$ & $l=6$ & $l=7$ & $l=8 \quad \sum_{l=0}^{k} E_{3}(k, l)$ \\
\hline 0 & 1 & 0 & 0 & 0 & 0 & 0 & 0 & 0 & 0 & $1=0 !$ \\
1 & 6 & 0 & 0 & 0 & 0 & 0 & 0 & 0 & 0 & $6=3 !$ \\
2 & 36 & 324 & 324 & 36 & 0 & 0 & 0 & 0 & 0 & $720=6 !$ \\
3 & 216 & 11664 & 87480 & 164160 & 87480 & 11664 & 216 & 0 & 0 & $362880=9 !$ \\
\hline
\end{tabular}


The first line for $k=0$ in Table 2 is not written in [1] [2] and then we have a triangle similar to the Pascal triangle and the notation $\left\langle\begin{array}{l}k \\ l\end{array}\right\rangle$ is chosen in analogy to the binomial coefficients $\left(\begin{array}{l}k \\ l\end{array}\right)$ in the Pascal triangle ${ }^{2}$

$$
\left(\begin{array}{l}
k \\
l
\end{array}\right)=\frac{k !}{l !(k-l) !},\left\langle\begin{array}{l}
k \\
l
\end{array}\right\rangle=\sum_{j=0}^{l} \frac{(-1)^{j}(k+1) !}{j !(k+1-j) !}(l+1-j)^{k} .
$$

With the line to $k=0$ in Table 2 the Eulerian numbers $E_{1}(k, l)$ form a triangle with an additional tip.

Similarly to the binomial coefficients in $(1+x)^{k}$, from the Tables $1-4$ we see that the polynomials $E_{\mu}(k ; z)$ are palindromical ones (for integer $\mu$ ) with the following relation for the coefficients $E_{\mu}(k, l)$ with fixed $k$

$$
E_{\mu}(k, l)=E_{\mu}(k, \mu(k-1)-l), \quad(k=0,1,2, \cdots),
$$

and for the coefficients in the first columns for $l=0$ one has

$$
E_{\mu}(k, 0)=\mu !^{k} .
$$

Obviously, as to see from (2.13) and from the tables, the numbers in all rows (fixed $k$ ) possess the common factor $\mu !^{k}$ in their factorization that is $1 !^{k}=1$ for the Eulerian numbers and in this sense the tables for $E_{\mu}(k, l)$ with $\mu \geq 2$ could be simplified. For the sum of all coefficients $E_{\mu}(k, l)$ over lone finds

$$
E_{\mu}(k ; z=1)=\sum_{l=0}^{\mu k} E_{\mu}(k, l)=(\mu k) !
$$

Although the properties (2.9), (2.10) and (2.11) are evident from Tables 1-4 and can be affirmed by computer up to higher values as given they have to be proved that we make in the following Sections.

The integers $k$ in the generalizations $G_{\mu}(k, l)$ of the Geometric series in (2.2) can be extended to arbitrary real numbers $k$ in the convergence region according to

$$
G_{\mu}(k ; z) \equiv \sum_{n=0}^{\infty}\left(\frac{(n+\mu) !}{n !}\right)^{k} z^{n} \equiv \frac{E_{\mu}(k ; z)}{(1-z)^{\mu k+1}} \equiv \frac{1}{(1-z)^{\mu k+1}} \sum_{l=0}^{\infty} E_{\mu}(k, l) z^{l},
$$

where then the polynomials $E_{\mu}(k ; z)$ make the transition to entire function with the infinite sequence of coefficients $E_{\mu}(k ; l)$ of their Taylor series given by (2.13)

$$
E_{\mu}(k, l) \equiv \sum_{j=0}^{l} \frac{(-1)^{j}(\mu k+1) !}{j !(\mu k+1-j) !}\left(\frac{(l+\mu-j) !}{(l-j) !}\right)^{k}, \quad(l=0,1,2, \cdots) .
$$

The functions $E_{\mu}(k ; z)$ are equal to the Taylor series of the functions $(1-z)^{\mu k+1} G_{\mu}(k ; z)$.

One may also interpolate between integer numbers $\mu$ by choosing for it real

${ }^{2} \mathrm{An}$ interesting analogy exists also to the Stirling numbers $S(k, l)$ of second kind (see Section 7). 
numbers. In this case one has to extend the summation over $z^{l}$ to infinity since we do not obtain an automatic restriction for $I$ from the formula (2.13) for the coefficients $E_{\mu}(k, l)$.

\section{Few Examples for Application of Generalized Eulerian Numbers}

We demonstrate in this Section the application of Eulerian numbers for the approximate evaluation of generalized Geometric series in cases where $k$ is not an integer and where the bounds of the sums become unrestricted.

First we consider the series $G_{1}(k, l)$ in (2.2) for two special cases of non-integer parameter $k$. For example, for the series $G_{1}(k, l)$ with $k=\frac{1}{2}$ one finds

$$
\begin{aligned}
G_{1}\left(\frac{1}{2} ; z\right) \equiv & \sum_{n=0}^{\infty} \sqrt{n+1} z^{n}=\frac{1}{(1-z)^{\frac{3}{2}}} \sum_{l=0}^{\infty} E_{1}\left(\frac{1}{2}, l\right) z^{l} \\
= & \frac{1}{(1-z)^{\frac{3}{2}}}\left\{1-\frac{1}{2}(3-2 \sqrt{2}) z-\frac{1}{8}(12 \sqrt{2}-3-8 \sqrt{3}) z^{2}\right. \\
& \left.-\frac{3}{16}(8 \sqrt{3}-11-2 \sqrt{2}) z^{3}+\cdots\right\} \\
= & \frac{1}{(1-z)^{\frac{3}{2}}}\left\{1-0.0857864 z-0.0142695 z^{2}-0.00524613 z^{3}+\cdots\right\}, \\
& \left(\sum_{l=0}^{\infty} E_{1}\left(\frac{1}{2}, l\right)=\left(\frac{1}{2}\right) !=\frac{\sqrt{\pi}}{2}=0.886227\right),
\end{aligned}
$$

where the polynomials $E_{1}(k ; z)$ become now infinite series, and with $k=-\frac{1}{2}$

$$
\begin{aligned}
G_{1}\left(-\frac{1}{2} ; z\right) \equiv & \sum_{n=0}^{\infty} \frac{z^{n}}{\sqrt{n+1}}=\frac{1}{(1-z)^{\frac{1}{2}}} \sum_{l=0}^{\infty} E_{1}\left(-\frac{1}{2}, l\right) z^{l} \\
= & \frac{1}{(1-z)^{\frac{1}{2}}}\left\{1+\frac{1}{2}(\sqrt{2}-1) z+\frac{1}{24}(8 \sqrt{3}-3-6 \sqrt{2}) z^{2}\right. \\
& \left.+\frac{1}{48}(21-3 \sqrt{2}-8 \sqrt{3}) z^{3}+\cdots\right\} \\
= & \frac{1}{(1-z)^{\frac{1}{2}}}\left\{1+0.2071068 z+0.0987969 z^{2}+0.0604365 z^{3}+\cdots\right\}, \\
& \left(\sum_{l=0}^{\infty} E_{1}\left(-\frac{1}{2}, l\right)=\left(-\frac{1}{2}\right) !=\sqrt{\pi}=1.77245\right) .
\end{aligned}
$$

The sum check of the coefficients shows that the first 4 terms of the approximation in (3.2) do not give for $|z| \rightarrow 1$ such a good convergence as it was obtained in (3.1) with the first 4 sum terms (0.894698 in (3.1) and 1.366340 in (3.2)). Using the given formulae it is easy to calculate by a computer the sum of coefficients up to high approximations and we can see the convergence to the 
values $\frac{\sqrt{\pi}}{2}$ and $\sqrt{\pi}$, respectively.

For $k=-1$ one obtains the following series with the known exact evaluation

$$
G_{1}(-1 ; z)=\sum_{n=0}^{\infty} \frac{z^{n}}{n+1}=-\frac{\log (1-z)}{z}=E_{1}(-1 ; z) .
$$

The sum converges for $|z|<1$ and the functions $G_{1}(k ; z)$ and $E_{1}(k ; z)$ become identical for $k=-1$.

As a further example we consider the sum $G_{2}\left(\frac{1}{2} ; z\right)$ with the coefficients $E_{2}\left(\frac{1}{2}, l\right)$ of $E_{2}\left(\frac{1}{2} ; z\right)=\sum_{l=0}^{\infty} E_{2}\left(\frac{1}{2}, l\right) z^{l}$ given in (3) and find $\left(E_{2}(k ; z)\right.$ becomes now an infinite series)

$$
\begin{aligned}
G_{2}\left(\frac{1}{2} ; z\right) \equiv & \sum_{n=0}^{\infty} \sqrt{(n+2)(n+1)} z^{n}=\frac{1}{(1-z)^{2}} \sum_{l=0}^{\infty} E_{2}\left(\frac{1}{2}, l\right) z^{l} \\
= & \frac{1}{(1-z)^{2}}\left\{\sqrt{2}-(2 \sqrt{2}-\sqrt{6}) z-(2 \sqrt{6}-\sqrt{2}-2 \sqrt{3}) z^{2}\right. \\
& \left.-(4 \sqrt{3}-\sqrt{6}-2 \sqrt{5}) z^{3}-\cdots\right\} \\
= & \frac{1}{(1-z)^{2}}\left\{1.4142136-0.3789374 z-0.0206643 z^{2}-0.0065775 z^{3}-\cdots\right\}, \\
& \left(\sum_{l=0}^{\infty} E_{2}\left(\frac{1}{2}, l\right)=1 !=1\right) .
\end{aligned}
$$

This formula provides usable approximations for the infinite sum on the left-hand side. The sum check for the first 4 coefficients gives the value 1.008034 and shows that it is already "relatively" near to the theoretical value 1 for the sum of the infinite number of coefficients.

The separated function $(1-z)^{-(\mu k+1)}$ from $G_{\mu}(k ; z)$ depends only on the product $\mu k$ of the parameters but not on $\mu$ and $k$ separately. By $\mu$-fold differentiation of the Geometric series one obtains

$$
\sum_{n=0}^{\infty} \frac{(n+\mu) !}{n !} z^{n}=\frac{\mu !}{(1-z)^{\mu+1}} \equiv G_{\mu}(1 ; z)=\frac{1}{(1-z)^{\mu+1}} \sum_{l=0}^{\mu} E_{\mu}(1, l) z^{l} .
$$

According to (3.13) the coefficients $E_{\mu}(1, l)$ can be calculated by

$$
\begin{aligned}
& E_{\mu}(1, l)=\sum_{j=0}^{l} \frac{(-1)^{j}(\mu+1) !(\mu+l-j) !}{j !(\mu+1-j) !(l-j) !}=\mu ! \delta_{l, 0}, \quad \sum_{l=0}^{\mu} E_{\mu}(1, l)=\mu !, \\
& E_{\mu}(1,0)=\mu !, \quad E_{\mu}(1, l)=0, \quad(l=1,2, \cdots),
\end{aligned}
$$

where the explicit result can be taken from comparison with (3.5). This case corresponds to $k=1$ and therefore $\mu k=\mu$ in the general formula for $G_{\mu}(k ; z)$ and the sum $\sum_{l=0}^{\mu k} E_{\mu}(k, l)=(\mu k)$ ! over the coefficients is here obviously confirmed. We assumed in the last derivation that $\mu$ is a non-negative integer but using fractional differentiation the result (3.5) is also correct if $\mu$ is an arbitrary non-negative number where, however, the coefficients $E_{\mu}(1, l)$ are 
not automatically vanishing for $l \geq 1$ and have to be taken into account in the sums.

\section{Proof of the General Relation for $E_{\mu}(k ; z)$ and Its Inversion}

We prove in this Section the general formula (2.4) for the functions $G_{\mu}(k ; z)$ together with the formulae (2.5) for the Eulerian polynomials $E_{\mu}(k ; z)$ with their coefficients $E_{\mu}(k ; l)$ in (2.13) and derive some consequences. For this purpose we multiply $G_{\mu}(k ; z)$ with $(1-z)^{\mu k+1}$ and apply for this factor the binomial formula and make a transformation by reordering of the sum terms of this product by substitution $j+n \rightarrow l$ in the arising double sum as follows

$$
\begin{aligned}
E_{\mu}(k ; z) & \equiv(1-z)^{\mu k+1} G_{\mu}(k ; z) \\
& \left.=\sum_{j=0}^{\infty} \sum_{n=0}^{\infty} \frac{(-1)^{j}(\mu k+1) !((\mu k+1-j) !}{j !(n+\mu) !}\right)^{k} z^{j+n} \\
& =\sum_{l=0}^{\infty}\left(\sum_{j=0}^{l} \frac{(-1)^{j}(\mu k+1) !}{j !(\mu k+1-j) !}\left(\frac{(l-j+\mu) !}{(l-j) !}\right)^{k}\right) z^{l} \\
& \equiv \sum_{l=0}^{\infty} E_{\mu}(k, l) z^{l} .
\end{aligned}
$$

Using the uniqueness of the Taylor series we find immediately in this way the formula (2.13) for the Generalized Eulerian numbers $E_{\mu}(k, l)$ as the coefficients in the expansion in powers $x^{l}$ that means by substitution of the summation index

$$
E_{\mu}(k, l)=\sum_{n=0}^{l} \frac{(-1)^{n-l}(\mu k+1) !}{(l-n) !(\mu k+1-l+n) !}\left(\frac{(n+\mu) !}{n !}\right)^{k} .
$$

As integration limit in the first summation over the functions $z^{l}$ in (4.1) we wrote $l \rightarrow \infty$ since the formula for $E_{\mu}(k, l)$ provides automatically the restriction $0 \leq l \leq \mu k+1$ in case that $\mu k$ is an integer and admits the useful extension to $0 \leq l \rightarrow \infty$ in case that $\mu k$ is an arbitrary real number.

We now derive the inversion of the transformation (4.2) that means we express $\left(\frac{(n+\mu) !}{n !}\right)^{k}$ by the Generalized Eulerian numbers $E_{\mu}(k, l)$. For this purpose we use the sum evaluation (4.4) with the split factor $\frac{1}{(1-z)^{\mu k+1}}$ and make a Taylor series expansion of this factor according to

$$
\begin{aligned}
\sum_{n=0}^{\infty}\left(\frac{(n+\mu) !}{n !}\right)^{k} z^{n} & =\frac{1}{(1-z)^{\mu k+1}} E_{\mu}(k ; z) \\
& =\sum_{j=0}^{\infty} \sum_{l=0}^{\mu k} \frac{(j+\mu k) !}{j !(\mu k) !} E_{\mu}(k, l) z^{j+l} \\
& =\sum_{n=0}^{\infty}\left(\sum_{j=0}^{\mu k} \frac{(j+\mu k) !}{j !(\mu k) !} E_{\mu}(k, n-j)\right) z^{n} .
\end{aligned}
$$


Thus we find the new identity

$$
\begin{aligned}
\left(\frac{(n+\mu) !}{n !}\right)^{k} & =\sum_{j=n-\mu k}^{n} \frac{(j+\mu k) !}{j !(\mu k) !} E_{\mu}(k, n-j) \\
& =\sum_{l=0}^{\mu k} \frac{(n-l+\mu k) !}{(n-l) !(\mu k) !} E_{\mu}(k, l) .
\end{aligned}
$$

In the special case $\mu=1$ we specialize from (4.4)

$$
(n+1)^{k}=\sum_{l=0}^{k} \frac{(n-l+k) !}{(n-l) ! k !} E_{1}(k, l)
$$

where $E_{1}(k, l) \equiv\left\langle\begin{array}{l}k \\ l\end{array}\right\rangle$ are the Eulerian numbers. The relation (4.5) is called the Worpitzky identity [2] (Petersen, p. 11 there).

\section{Recurrence Relation $k \rightarrow k+1$ for $G_{\mu}(k ; z)$ and $E_{\mu}(k ; z)$}

We now derive a recurrence relation for $k \rightarrow k+1$ of $G_{\mu}(k ; z)$. For this purpose we multiply first $G_{\mu}(k ; z)$ with $z^{\mu}$ and form the the $\mu$-th derivative of this product

$$
\begin{aligned}
\frac{\partial^{\mu}}{\partial z^{\mu}} z^{\mu} G_{\mu}(k ; z) & =\frac{\partial^{\mu}}{\partial z^{\mu}} \sum_{n=0}^{\infty}\left(\frac{(n+\mu) !}{n !}\right)^{k} z^{n+\mu} \\
& =\sum_{n=0}^{\infty}\left(\frac{(n+\mu) !}{n !}\right)^{k+1} z^{n}=G_{\mu}(k+1 ; z) .
\end{aligned}
$$

Thus we have already obtained the required recurrence relation. The operator $\frac{\partial^{\mu}}{\partial z^{\mu}} z^{\mu}$ is in analogy to anti-normal ordering and in quantum optics of the boson annihilation and creation operators $\left(a, a^{\dagger}\right)$ it is known how one can bring this to normal ordering (e.g., [8]; can be proved by complete induction; see Appendix A), in application to our case

$$
G_{\mu}(k+1 ; z)=\frac{\partial^{\mu}}{\partial z^{\mu}} z^{\mu} G_{\mu}(k ; z)=\sum_{j=0}^{\mu} \frac{\mu !^{2}}{j !(\mu-j) !^{2}} z^{\mu-j} \frac{\partial^{\mu-j}}{\partial z^{\mu-j}} G_{\mu}(k ; z) .
$$

Both forms of operator ordering are appropriate for the following calculations but anti-normal ordering proved to be here more simple for us.

Using the connection (5.4) of $G_{\mu}(k ; z)$ to the Generalized Eulerian polynomials $E_{\mu}(k ; z)$ we find in anti-normal ordering

$$
\frac{E_{\mu}(k+1 ; z)}{(1-z)^{\mu(k+1)+1}}=\frac{\partial^{\mu}}{\partial z^{\mu}} z^{\mu} \frac{E_{\mu}(k ; z)}{(1-z)^{\mu k+1}} .
$$

We multiply this identity with $(1-z)^{\mu(k+1)+1}$ and bring the right-hand side to anti-normal ordering using the commutation rules (5.1) of Appendix A and then the disentanglement relation (5.3) to anti-normal ordering (see Appendix B) 


$$
\begin{aligned}
E_{\mu}(k+1 ; z) & =(1-z)^{\mu(k+1)+1} \frac{\partial^{\mu}}{\partial z^{\mu}} z^{\mu} \frac{E_{\mu}(k ; z)}{(1-z)^{\mu k+1}} \\
& =\sum_{i=0}^{\mu} \frac{\mu !(\mu(k+1)+1) !}{i !(\mu-i) !(\mu k+1+i) !}\left(\frac{\partial^{i}}{\partial z^{i}} z^{\mu}(1-z)^{i}\right) E_{\mu}(k ; z) .
\end{aligned}
$$

Here we make two remarks. From (see (2.5))

$$
E_{\mu}(k ; z)=\sum_{l=0}^{\mu k} E_{\mu}(k, l) z^{l},
$$

first follows

$$
E_{\mu}(k, l)=\frac{1}{l !}\left\{\frac{\partial^{l}}{\partial z^{l}} E_{\mu}(k ; z)\right\}_{z=0} \Rightarrow E_{\mu}(k+1, l)=\frac{1}{l !}\left\{\frac{\partial^{l}}{\partial z^{l}} E_{\mu}(k+1 ; z)\right\}_{z=0},
$$

and second, the already mentioned

$$
\left\{E_{\mu}(k ; z)\right\}_{z=1}=\sum_{l=0}^{\mu k} E_{\mu}(k, l),
$$

that means the sum of coefficients $E_{\mu}(k, l)$ in each row with fixed $k$ in the tables of the Generalized Eulerian polynomials $E_{\mu}(k ; z)$.

If we insert (5.5) into (5.4) we find

$$
\begin{aligned}
E_{\mu}(k+1 ; z) & =\sum_{l=0}^{\mu(k+1)} E_{\mu}(k+1, l) z^{l} \\
& =\sum_{i=0}^{\mu} \frac{\mu !(\mu(k+1)+1) !}{i !(\mu-i) !(\mu k+1+i) !} \sum_{m=0}^{\mu k}\left(\frac{\partial^{i}}{\partial z^{i}} z^{\mu+m}(1-z)^{i}\right) E_{\mu}(k, m) .
\end{aligned}
$$

To get the recurrence relation for $E_{\mu}(k+1, l)$ we have according to (5.6) and using (5.8) to form

$$
\begin{aligned}
& E_{\mu}(k+1, l) \\
& =\frac{1}{l !}\left\{\frac{\partial^{l}}{\partial z^{l}} \sum_{i=0}^{\mu} \frac{\mu !(\mu(k+1)+1) !}{i !(\mu-i) !(\mu k+1+i) !} \sum_{m=0}^{\mu k}\left(\frac{\partial^{i}}{\partial z^{i}} z^{\mu+m}(1-z)^{i}\right) E_{\mu}(k, m)\right\}_{z=0} \\
& =\frac{1}{l !} \sum_{i=0}^{\mu} \frac{\mu !(\mu(k+1)+1) !}{i !(\mu-i) !(\mu k+1+i) !} \sum_{m=0}^{\mu k}\left\{\frac{\partial^{l+i}}{\partial z^{l+i}} z^{\mu+m}(1-z)^{i}\right\}_{z=0} E_{\mu}(k, m) .
\end{aligned}
$$

The detailed calculation in Appendix A using the general formula for the inner derivative (A1) at $z=0$ together with (A2) leads in (5.9) to the general recurrence relation for $E_{\mu}(k+1, l)$

$$
\begin{aligned}
& E_{\mu}(k+1, l) \\
& =\frac{\mu !(\mu(k+1)+1) !}{l !} \sum_{i=0}^{\mu} \frac{(-1)^{i}(l+i) !}{i !(\mu-i) !(\mu k+1+i) !} \sum_{j=0}^{i} \frac{(-1)^{j} i !}{j !(i-j) !} E_{\mu}(k, l-\mu+j) \\
& =\sum_{n=0}^{\mu} \frac{\mu !}{n !(\mu-n) !}\left(\sum_{m=0}^{n} \frac{(-1)^{n-m} n !}{m !(n-m) !} \frac{(\mu(k+1)+1) !}{(\mu(k+1)+1-m) !} \frac{(l+\mu-m) !}{l !}\right) E_{\mu}(k, l-n),
\end{aligned}
$$

where we used a substitution of the summation variable. The inner sum in (5.10) can be evaluated using the Jacobi polynomials and we find in different 
compositions of the factorials to binomial coefficients

$$
\begin{aligned}
E_{\mu}(k+1, l) & =\sum_{n=0}^{\mu} \frac{\mu !}{n !(\mu-n) !} \frac{(l+\mu-n) !(n+\mu k-l) !}{l !(\mu k-l) !} E_{\mu}(k, l-n) \\
& =\mu ! \sum_{n=0}^{\mu} \frac{(l+\mu-n) !}{l !(n-n) !} \frac{(n+\mu k-l) !}{n !(\mu k-l) !} E_{\mu}(k, l-n),
\end{aligned}
$$

or equivalently in the form (for later use)

$$
E_{\mu}(k+1, l)=\sum_{m=l-\mu}^{l} \frac{\mu !(\mu k-m) !(m+\mu) !}{l !(\mu k-l) !(m+\mu-l) !(l-m) !} E_{\mu}(k, m) .
$$

Thus the recurrence relations possess $\mu+1$ sum terms on the right-hand side proportional to the numbers $E_{\mu}(k, l), E_{\mu}(k, l-1), \cdots, E_{\mu}(k, l-\mu)$.

For the first four special cases $\mu=0,1,2,3$ we find from (5.10) or (5.11)

$$
\begin{aligned}
E_{0}(k+1, l)= & E_{0}(k, l), \\
E_{1}(k+1, l)= & (l+1) E_{1}(k, l)+(k-l+1) E_{1}(k, l-1), \\
E_{2}(k+1, l)= & (l+2)(l+1) E_{2}(k, l)+2(2 k-l+1)(l+1) E_{2}(k, l-1) \\
& +(2 k-l+2)(2 k-l+1) E_{2}(k, l-2), \\
E_{3}(k+1, l)= & (l+3)(l+2)(l+1) E_{3}(k, l) \\
& +3(3 k-l+1)(l+2)(l+1) E_{3}(k, l-1) \\
& +3(3 k-l+2)(3 k-l+1)(l+1) E_{3}(k, l-2) \\
& +(3 k-l+3)(3 k-l+2)(3 k-l+1) E_{3}(k, k-3),
\end{aligned}
$$

The second of these relations which written by $\left\langle\begin{array}{l}k \\ l\end{array}\right\rangle \equiv E_{1}(k, l)$ takes on the form

$$
\left\langle\begin{array}{c}
k+1 \\
l
\end{array}\right\rangle=(l+1)\left\langle\begin{array}{l}
k \\
l
\end{array}\right\rangle+(k+1-l)\left\langle\begin{array}{c}
k \\
l-1
\end{array}\right\rangle,
$$

is the recurrence relation for the Eulerian numbers with a certain similarity to the recurrence relation for the binomial coefficients $\left(\begin{array}{l}k \\ l\end{array}\right)$ and as we later see to that for Stirling numbers of second kind. It is known (Riordan [1], chap. 8, Petersen [2], p. 8, Equation (1.7), Bressoud, p. 632).

In the representation (5.12) of $E_{\mu}(k+1, l)$ we may sum over $l$ using the Vandermonde's convolution identity $\sum_{l=0}^{n}\left(\begin{array}{l}n \\ l\end{array}\right)\left(\begin{array}{l}c-n \\ a-l\end{array}\right)=\left(\begin{array}{l}c \\ a\end{array}\right)$ or in the form

$$
\sum_{l=0}^{\{\mu, v\}} \frac{\mu ! v !}{l !(\mu-l) !(v-l) !(l+\lambda) !}=\frac{(\mu+v+\lambda) !}{(\mu+\lambda) !(v+\lambda) !},
$$

and one obtains the following recurrence relation for the sums over all coefficients $E_{\mu}(k, l)$ for fixed $k$

$$
\sum_{l=0}^{\mu(k+1)} E_{\mu}(k+1, l)=\frac{(\mu(k+1)) !}{(\mu k) !} \sum_{m=0}^{\mu k} E_{\mu}(k, m) .
$$


This recurrence relation with the initial condition $\sum_{l=0}^{0} E_{\mu}(1, l)=\mu$ ! (or $\sum_{l=0}^{0} E_{\mu}(0, l)=1$ !) is solved by

$$
\sum_{l=0}^{\mu k} E_{\mu}(k, l)=(\mu k) !
$$

as can be proved by complete induction. There are other possibilities to prove this equation (see next Section) but from the explicit expression for $E_{\mu}(k, l)$ given in (2.13) we could not find a simple direct proof of this result.

\section{Recurrence Relation $\mu \rightarrow \mu+1$ for $G_{\mu}(k ; z)$ and}

$$
E_{\mu}(k ; z)
$$

We now derive a recurrence relation $\mu \rightarrow \mu+1$ for $G_{\mu}(k ; z)$ by applying the operator $\left(z \frac{\partial}{\partial z}\right)^{k}$ to $G_{\mu}(k ; z)$ using the eigenvalue property

$$
\begin{aligned}
\left(z \frac{\partial}{\partial z}\right)^{k} z^{n}=n^{k} z^{n} & \\
\left(z \frac{\partial}{\partial z}\right)^{k} G_{\mu}(k ; z) & \equiv\left(z \frac{\partial}{\partial z}\right)^{k} \sum_{n=0}^{\infty}\left(\frac{(n+\mu) !}{n !}\right)^{k} z^{n} \\
& =\sum_{n=0}^{\infty}\left(\frac{(n+\mu) !}{(n-1) !}\right)^{k} z^{n}=\sum_{m=0}^{\infty}\left(\frac{(m+\mu+1) !}{m !}\right)^{k} z^{m+1} \\
& \equiv z G_{\mu+1}(k ; z), \quad(k \neq 0) .
\end{aligned}
$$

The case $k=0$ makes an exception from this recurrence relation due to

$$
G_{\mu}(0 ; z)=\sum_{n=0}^{\infty} z^{n}=\frac{1}{1-z}=G_{\mu+1}(0 ; z) .
$$

We apply now the transition to normal ordering. The operator $\left(z \frac{\partial}{\partial z}\right)^{k}$ in (6.1) can be "disentangled" using the Stirling numbers of second kind $S(k, l)$ (e.g., [1] [3] [9]) in the following way (see Appendix C)

$$
\left(z \frac{\partial}{\partial z}\right)^{k}=\sum_{j=0}^{k} S(k, j) z^{j} \frac{\partial^{j}}{\partial z^{j}} .
$$

This can be proved by complete induction using the known recurrence relations for the Stirling numbers of second kind (see Appendix C). Applying the relation (6.4) of $G_{\mu}(z ; k)$ to the Generalized Eulerian polynomials $E_{\mu}(k ; z)$ from (6.1) follows

$$
\begin{aligned}
\frac{z E_{\mu+1}(k ; z)}{(1-z)^{(\mu+1) k+1}} & =\sum_{j=0}^{k} S(k, j) z^{j} \frac{\partial^{j}}{\partial z^{j}} \frac{E_{\mu}(k ; z)}{(1-z)^{\mu k+1}} \\
& =\frac{1}{(1-z)^{\mu k+1}} \sum_{j=0}^{k} S(k, j) z^{j}\left(\frac{\partial}{\partial z}+\frac{\mu k+1}{1-z}\right)^{j} E_{\mu}(k ; z) .
\end{aligned}
$$

Using the auxiliary formula (6.7) in the second form this can be transformed to 


$$
\begin{aligned}
& z E_{\mu+1}(k ; z)=(1-z)^{k} \sum_{j=0}^{k} S(k, j) z^{j} \sum_{i=0}^{j} \frac{j !(\mu k+i) !}{i !(j-i) !(\mu k) !} \frac{1}{(1-z)^{i}} \frac{\partial^{j-i}}{\partial z^{j-i}} E_{\mu}(k ; z) \\
& =\frac{1}{(\mu k) !} \sum_{l=0}^{\mu k} \frac{1}{l !}\left(\sum_{j=0}^{k} S(k, j) z^{j} \frac{j !(\mu k+j-l) !}{(j-l) !}(1-z)^{k+l-j}\right) \frac{\partial^{l}}{\partial z^{l}} E_{\mu}(k ; z) \\
& =\frac{1}{(\mu k) !} \sum_{m=0}^{\mu k} E_{\mu}(k, m)\left(\sum_{l=0}^{m} \frac{m !}{l !(m-l) !} \sum_{j=0}^{k} S(k, j) \frac{j !(\mu k+j-l) !}{(j-l) !} z^{j+m-l}(1-z)^{k+l-j}\right),
\end{aligned}
$$

with exclusion of the case $k=0 \quad$ (see (6.2)).

First we consider the recurrence relation for the sum of the coefficients $E_{\mu}(k, l)$ over 1 . From

$$
\begin{aligned}
& \sum_{l=0}^{(\mu+1) k} E_{\mu+1}(k, l) \\
= & E_{\mu+1}(k ; z=1)=\lim _{z \rightarrow 1}\left(z E_{\mu+1}(k ; z)\right),
\end{aligned}
$$

applied to the right-hand side of (6.5) follows that it can be different from zero only if $\lim _{z \rightarrow 1}(1-z)^{k+l-j}$ is different from zero that is only the case if $k+l-j=0$ and we find

$$
\begin{aligned}
& \sum_{l=0}^{(\mu+1) k} E_{\mu+1}(k, l) \\
= & \frac{1}{(\mu k) !} \sum_{m=0}^{\mu k} E_{\mu}(k, m) \sum_{l=0}^{m} \underbrace{S(k, k+l)}_{=\delta_{l, 0}} \frac{m !}{l !(m-l) !} \frac{(k+l) !((\mu+1) k) !}{k !} \\
= & \frac{((\mu+1) k) !}{(\mu k) !} \sum_{m=0}^{\mu k} E_{\mu}(k, m) .
\end{aligned}
$$

To get the second line we used that $S(k, k+l)$ is only different from zero for $l=0$ and $S(k, k)=1$. In this way we found the recurrence relation for the sums $\sum_{l=0}^{\mu k} E_{\mu}(k, l)$ from $\mu \rightarrow \mu+1$ which with

$$
\sum_{l=0}^{0} E_{0}(k, l)=1, \quad \sum_{l=0}^{k} E_{1}(k, l)=k !
$$

leads to the already known solution (5.17).

We now try to derive a recurrence relation for the coefficients $E_{\mu}(k, l)$ from $\mu \rightarrow \mu+1$ and mention for this purpose

$$
E_{\mu+1}(k, l)=\frac{1}{l !} \lim _{z \rightarrow 0} \frac{\partial^{l}}{\partial z^{l}} E_{\mu+1}(k ; z)=\frac{1}{l !}\left\{\frac{\partial^{l}}{\partial z^{l}} \sum_{m=0}^{(\mu+1) k} E_{\mu+1}(k, m) z^{m}\right\}_{z=0} .
$$

From the Leibniz formula for the multiple derivatives of a product of two functions

$$
\frac{\partial^{l}}{\partial z^{l}} f(z) g(z)=\sum_{k=0}^{l} \frac{l !}{k !(l-k) !} f^{(k)}(z) g^{(l-k)}(z),
$$

one derives the following auxiliary formula 


$$
\begin{aligned}
& \left\{\frac{\partial^{l}}{\partial z^{l}} z^{p}(1-z)^{q}\right\}_{z=0} \\
& =\left\{\sum_{k=0}^{l} \frac{l !}{k !(l-k) !} \frac{p !}{(p-k) !} z^{p-k} \frac{(-1)^{l-k} q !}{(q-l+k) !}(1-z)^{q-l+k}\right\}_{z=0} \\
& =\frac{(-1)^{l-p} l ! q !}{(l-p) !(q-l+p) !} .
\end{aligned}
$$

It is different from zero only for $0 \leq l-p \leq q$.

We now calculate the recurrence relation for $E_{\mu}(k, l)$ from $\mu \rightarrow \mu+1$. Since according to (6.9) we have to apply the operator $\frac{\partial^{l}}{\partial z^{l}}$ to $E_{\mu}(k ; z)$ we have first to change the summation index $l$ on the right-hand side in (6.5) and write this relation in the form

$$
\begin{aligned}
E_{\mu+1}(k, z)= & \frac{1}{(\mu k) !} \sum_{m=0}^{\mu k} E_{\mu}(k, m) \sum_{j=0}^{k} S(k, j) j ! \\
& \cdot \sum_{n=0}^{m} \frac{m !(\mu k+j-n) !}{n !(m-n) !(j-n) !}\left(z^{j+m-n-1}(1-z)^{k-j+n}\right),
\end{aligned}
$$

where we also changed the order of the inner summations that, however, is subsidiary. Now we may apply the auxiliary identity (6.11) when we accomplish the differentiations by the operator $\frac{1}{l !}\left\{\frac{\partial^{l}}{\partial z^{l}} \ldots\right\}_{z=0}$ in (6.9). With corresponding substitutions this led us to the following recurrence relation with a triple sum

$$
\begin{aligned}
E_{\mu+1}(k, l)= & \frac{(-1)^{l+1}}{(\mu k) !} \sum_{m=0}^{\mu k} E_{\mu}(k, m) \frac{(-1)^{m} m !}{(k+m-l-1) !} \sum_{j=0}^{k} S(k, j) j ! \\
& \cdot \sum_{n=0}^{\{j, m\}} \frac{(-1)^{j-n}(\mu k+j-n) !(k-j+n) !}{n !(j-n) !(m-n) !(l-j-m+1+n) !}, \quad(k \neq 0) .
\end{aligned}
$$

We checked by computer the correctness of formulae (6.13) and also of (6.5) in comparison to the formula (6.13). If we represent (6.13) in the form

$$
E_{\mu+1}(k, l) \equiv \sum_{m=0}^{\mu k} f_{\mu}(k, l, m) E_{\mu}(k, m),
$$

where the numbers $f_{\mu}(k, l, m)$ are given by a double sum which can be taken from (6.13) then by prime-number analysis of $f_{\mu}(k, l, m)$ we find that for some relatively low integers $(k, l, m)$ we have already in some cases relatively high prime numbers and it is unlikely that one can find a closed simple formula of multiplicative type for these coefficients. It seems to us also unlikely that one may find simple intermediate results for the evaluation of one of the double sums in $f_{\mu}(k, l, m)$ such as given in (6.13) or by possible reordering of terms of this double sum. Nevertheless, this is astonishing in view of the simple result (6.13) for $E_{\mu}(k, l)$.

We mention that the inner sum in (6.13) can be represented as polynomial case of the Hypergeometric function ${ }_{3} \mathrm{~F}_{2}\left(a_{1}, a_{2}, a_{3} ; c_{1}, c_{2} ; z\right)$ with special 
argument $Z=1$ as follows (can be directly taken from Wolfram's "Mathematica" but is also not so difficult to specialize from the mentioned Hypergeometric function in a transformed form)

$$
\begin{aligned}
& \sum_{n=0}^{\{j, m\}} \frac{(-1)^{j-n}(\mu k+j-n) !(k-j+n) !}{n !(j-n) !(m-n) !(l-j-m+1+n) !} \\
& \equiv \frac{(-1)^{j}(\mu k+j) !(k-j) !}{j !(l-j-m+1) !}{ }_{3} \mathrm{~F}_{2}(-j,-m, k+l-j ; l-j-m+2,-\mu k-j ; 1),
\end{aligned}
$$

with 5 parameters $(\mu ; j, k, l, m)$. This sum is different from zero only for

$$
\begin{aligned}
& 0 \leq j-n \leq l+1-m \Leftrightarrow m \leq j+m-n \leq l+1, \\
& 0 \leq m-n \leq l+1-j \Leftrightarrow j \leq j+m-n \leq l+1 .
\end{aligned}
$$

We see that all coefficients $E_{\mu}(k, m)$ with $m \leq l+1$ are involved in the recurrence relation (6.14). The only known nontrivial sum identity for ${ }_{3} \mathrm{~F}_{2}(\ldots)$, the Pfaff-Saalschütz identity (see Andrews, Askey and Roy [10], pp. 69, 70) cannot be fitted to the present case.

All this shows that the recurrence relation (6.13) from $\mu \rightarrow \mu+1$ is probably of limited value for analysis in comparison to the recurrence relation from $k \rightarrow k+1$ with fixed $\mu$ which is given in (5.10) and specialized in (5.13).

\section{Relation between Eulerian Numbers and Stirling Numbers of Second Kind}

In this Section we give proofs for relations of the Eulerian numbers to the Stirling numbers of second kind and of their inversions.

If we compare the explicit representations of the Stirling numbers of second kind $S(k, l)$ with that of the Eulerian numbers $E_{1}(k, l)$

$$
\begin{aligned}
& S(k, l)=\sum_{j=0}^{l} \frac{(-1)^{j}}{j !(l-j) !}(l-j)^{k}, \quad S(0,0)=1, \\
& E_{1}(k, l)=\sum_{j=0}^{l} \frac{(-1)^{j}(k+1) !}{j !(k+1-j) !}(l+1-j)^{k} \equiv\left\langle\begin{array}{l}
k \\
l
\end{array}\right\rangle,
\end{aligned}
$$

then we see certain similarities. The same is between the representations of $(n+1)^{k}$ by Stirling numbers of second kind according to

$$
(n+1)^{k}=\sum_{l=0}^{k}(-1)^{k-l} S(k, l) \frac{(n+l) !}{n !}=\sum_{l=0}^{k} S(k+1, l+1) \frac{n !}{(n-l) !},
$$

and by Eulerian numbers according to

$$
(n+1)^{k}=\sum_{l=0}^{k-1} E_{1}(k, l) \frac{(n+k-l) !}{k !(n-l) !} .
$$

We now derive an expression of the Eulerian numbers $E_{1}(k, l)$ by the Stirling numbers of second kind $S(n, k)$.

For our objective we consider the definition (2.2) for $G_{1}(k ; z)$ and after using the first of the relations (7.2) we make a reordering of the arising double sum 


$$
\begin{aligned}
\sum_{n=0}^{\infty}(n+1)^{k} z^{n} & =\sum_{n=0}^{\infty} z^{n} \sum_{j=0}^{k}(-1)^{k-j} S(k, j) \frac{(n+j) !}{n !} \\
& =\sum_{j=0}^{k}(-1)^{k-j} S(k, j) \sum_{n=0}^{\infty} \frac{(n+j) !}{n !} z^{n} \\
& =\sum_{j=0}^{k}(-1)^{k-j} S(k, j) \frac{j !}{(1-z)^{j+1}},
\end{aligned}
$$

where we have evaluated the inner sum. Now we split a function $\frac{1}{(1-z)^{k+1}}$ from the last result and expand the remaining function in a Taylor series with respect to powers $z^{l}$

$$
\begin{aligned}
\sum_{n=0}^{\infty}(n+1)^{k} z^{n} & =\frac{1}{(1-z)^{k+1}} \sum_{j=0}^{k}(-1)^{k-j} S(k, j) j !(1-z)^{k-j} \\
& =\frac{1}{(1-z)^{k+1}} \sum_{l=0}^{k}\left(\sum_{j=0}^{k-l}(-1)^{k-j-l} S(k, j) \frac{j !(k-j) !}{l !(k-j-l) !}\right) z^{l} .
\end{aligned}
$$

By comparison with

$$
\sum_{n=0}^{\infty}(n+1)^{k} z^{n}=\frac{1}{(1-z)^{k+1}} \sum_{l=0}^{k} E_{1}(k, l) z^{l},
$$

follows for the relation between Eulerian numbers $E_{1}(k, l)$ and Stirling numbers of second kind $S(k, l) \quad$ [3] (Eq. 26.14.7)

$$
E_{1}(k, l)=\frac{(-1)^{k-l}}{l !} \sum_{j=0}^{k-l}(-1)^{j} \frac{j !(k-j) !}{(k-l-j) !} S(k, j) .
$$

The inversion of this relation is [3] (Eq. 26.14.12)

$$
S(k, l)=\frac{1}{l !} \sum_{i=0}^{l} \frac{(k-l+i) !}{(k-l) ! i !} E_{1}(k, k-l+i) .
$$

This can be proved by inserting $S(k, j)$ according to (7.8) into (7.7) and reordering of the double sum

$$
\begin{aligned}
E_{1}(k, l) & =\frac{(-1)^{k-l}}{l !} \sum_{j=0}^{k-l}(-1)^{j} \frac{j !(k-j) !}{(k-l-j) !} \frac{1}{j !} \sum_{i=0}^{j} \frac{(k-j+i) !}{i !(k-j) !} E_{1}(k, k-j+i) \\
& =\frac{1}{l !} \sum_{m=0}^{k} E_{1}(k, m) \frac{(-1)^{m-l} m !}{(m-l) !} \underbrace{m-l}_{=(1-1)^{m-l}=\delta_{m, l}} \frac{(-1)^{i}(m-l) !}{i !(m-l-i) !}
\end{aligned}
$$

We see that the right-hand sides are equal to the left-hand sides for all $(k, l)$. If we use the second alternative representation of $(n+1)^{k}$ by the Stirling numbers of second kind one finds an alternative representation of $E_{1}(k, l)$ by $S(k, l)$. Instead of (4) we find then

$$
\sum_{n=0}^{\infty}(n+1)^{k} z^{n}=\sum_{j=0}^{k} S(k+1, j+1) \frac{j ! z^{j}}{(1-z)^{j+1}},
$$

and instead of (5) 


$$
\sum_{n=0}^{\infty}(n+1)^{k} z^{n}=\frac{1}{(1-z)^{k+1}} \sum_{l=0}^{k}\left(\sum_{j=0}^{l} S(k+1, j+1)(-1)^{l-j} \frac{j !(k-j) !}{(l-j) !(k-l) !}\right) z^{l} .
$$

By comparison with (7.6) we arrive at the following alternative representation of Eulerian numbers $E_{1}(k, l)$ by Stirling numbers of second kind $S(k, l)$

$$
E_{1}(k, l)=\frac{1}{(k-l) !} \sum_{j=0}^{l}(-1)^{l-j} \frac{j !(k-j) !}{(l-j) !} S(k+1, j+1) .
$$

The inversion of this relation is

$$
S(k+1, l+1)=\frac{1}{l !} \sum_{i=0}^{l} \frac{(k-l+i) !}{(k-l) ! i !} E_{1}(k, l-i) .
$$

One may be astonished that two differently looking representations (7.7) and (7.12) of the Eulerian numbers by the Stirling numbers of second kind are possible but one has the following relation between $S(k+1, l+1)$ and $S(k, l)$

$$
S(k+1, l+1)=\sum_{j=0}^{k} \frac{k !}{j !(k-j) !} S(j, l),
$$

with the inversion

$$
S(k, l)=\sum_{j=0}^{k}(-1)^{k-j} \frac{k !}{j !(k-j) !} S(j+1, l+1) .
$$

These relations can be proved using the recurrence relations for the Stirling numbers of second kind.

\section{Conclusion}

We derived in this paper properties of sets of numbers

$E_{\mu}(k, l),(\mu=0,1,2, \cdots, k=0,1,2, \cdots, l=0,1, \cdots, \mu l)$, which for $\mu=1$ are the Eulerian numbers and which for $\mu \geq 2$ could be called Generalized Eulerian numbers. A main purpose was to obtain sum approximation for some generalized Geometric series denoted by $G_{\mu}(k ; z)$ with extension of $k$ to arbitrary real numbers providing for $|z|<1 ; z \rightarrow 1$ that means for the convergence region of these series acceptable approximations for the sums taking into account a few number of corresponding terms with powers of $z$ in the Generalized Eulerian polynomials $E_{\mu}(k ; z)$ with non-integer $k$. We proposed notations for these generalizations since the notation $\left\langle\begin{array}{l}n \\ l\end{array}\right\rangle$ for the Eulerian numbers is difficult to extend. The total number $\sum_{l=0}^{\mu k} E_{\mu}(k, l)=(\mu k)$ ! suggests a combinatorial subdivision of the permutations of $\mu k$ elements in classes with certain mutually not intersecting properties for example, as it is possible and known for the Eulerian numbers $E_{1}(k, l)$.

In comparison to the Hypergeometric functions ${ }_{p} \mathrm{~F}_{q}\left(a_{1}, \cdots, a_{r} ; c_{1}, \cdots, c_{s} ; z\right)$ the series $G_{\mu}(k ; z)$ involving in their evaluation the numbers $E_{\mu}(k, l)$ represent a generalization of the Geometric series in a different direction. Considering the Hypergeometric functions with different orders of growth for 
increasing $z>0$ or with finite convergence radius such as the Geometric series

$$
\begin{gathered}
{ }_{1} \mathrm{~F}_{0}(1 ;-; z)=\sum_{n=0}^{\infty} z^{n}=\frac{1}{1-z} \Rightarrow \frac{\partial^{n}}{\partial z^{n}} \frac{1}{1-z}=\frac{n !}{(1-z)^{n+1}}, \\
{ }_{0} \mathrm{~F}_{0}(-;-; z)=\sum_{n=0}^{\infty} \frac{z^{n}}{n !}=\exp (z), \\
{ }_{0} \mathrm{~F}_{1}(-; 1 ; z)=\sum_{n=0}^{\infty} \frac{z^{n}}{n !^{2}}=\mathrm{I}_{0}(2 \sqrt{z}) \Rightarrow \frac{\partial^{n}}{\partial z^{n}} \mathrm{I}_{0}(2 \sqrt{z})=\frac{\mathrm{I}_{n}(2 \sqrt{z})}{(\sqrt{z})^{n}},
\end{gathered}
$$

we think that it is possible to generalize the second and third function in a similar way as we made this here for the Geometric series $\sum_{n=0}^{\infty} z^{n}$ since the derivatives of these functions are easy to obtain.

We mention that to problems which we see at this moment belong the calculation of generating functions for the numbers $E_{\mu}(k, l)$ and the extension of the connection of Stirling numbers $S(k, l)$ to Eulerian numbers from $E_{1}(k, l)$ onto the whole set of numbers $E_{\mu}(k, l)$. Furthermore, it would be interesting to establish the evident relation of the Generalized Eulerian numbers to problems of combinatorics.

\section{References}

[1] Riordan, J. (1958) An Introduction to Combinatorial Analysis. John Wiley \& Sons, New York.

[2] Petersen, T.K. (2015) Eulerian Numbers. Birkhäuser (Springer), New York. https://doi.org/10.1007/978-1-4939-3091-3

[3] Bressoud, D.M. (2010) Combinatorial Analysis. In: Olver, F.W.J., Lozier, D.W., Boisvert, R.F. and Clark, C.W., Eds., NIST Handbook of Mathematical Functions, NIST and Cambridge University Press, Cambridge, 617-636.

[4] Olver, F.W.J., Lozier, D.W., Boisvert, R.F. and Clark, C.W. (2010) NIST Handbook of Mathematical Functions. Cambridge University Press, Cambridge.

[5] Conway, J.H. and Guy, R.K. (1996) The Book of Numbers. Springer, New York.

[6] Weisstein, E.W. (2009) The CRC Encyclopedia of Mathematics. 3rd Edition, Chapman and Hall, CRC Press, Boca Raton.

[7] Wünsche, A. (2015) Quantum-Mechanical Cumulant Expansions and Their Application to Phase-Space and to Phase Distributions. Physica Scripta, 90, Article ID: 074063. https://doi.org/10.1088/0031-8949/90/7/074063

[8] Wünsche, A. (1999) Ordered Operator Expansions and Reconstruction from Ordered Moments. Journal of Optics B: Quantum and Semiclassical Optics, 1, 264-288. https://doi.org/10.1088/1464-4266/1/2/010

[9] Van Lint, J.H. and Wilson, R.M. (1992) A Course in Combinatorics. Cambridge University Press, Cambridge.

[10] Andrews, G.E., Askey, R. and Roy, R. (1999) Special Functions. Cambridge University Press, Cambridge. https://doi.org/10.1017/CBO9781107325937

[11] Wünsche, A. (2015) Operator Methods and SU (1,1) Symmetry in the Theory of Jacobi and of Ultraspherical Polynomials. Applied Mathematics, 7, 213-261.

[12] Gradshteyn, I.S. and Ryzhik, I.M. (1963) Tables of Series, Products and Integrals. 4th Edition, Nauka, Moskva. 


\section{Appendix A}

\section{Proof of the recurrence relations for Generalized Eulerian numbers}

In this Appendix we consider more in detail the derivation of the recurrence relations for the Generalized Eulerian numbers. In preparation we first derive an auxiliary formula which we later apply.

We need in the following the $l$-th derivatives of a product $z^{m}(1-z)^{n}$ taken at $z=0$

$$
\begin{aligned}
\left\{\frac{\partial^{l}}{\partial z^{l}} z^{m}(1-z)^{n}\right\}_{z=0} & =\sum_{k=0}^{n} \frac{(-1)^{k} n !}{k !(n-k) !}\left\{\frac{\partial^{l}}{\partial z^{l}} z^{k+m}\right\}_{z=0} \\
& =\sum_{k=0}^{n} \frac{(-1)^{k} n !}{k !(n-k) !} \frac{(k+m) !}{(k+m-l) !}\left\{z^{k+m-l}\right\}_{z=0} \\
& =\sum_{k=0}^{n} \frac{(-1)^{k} n !(k+m) !}{k !(n-k) !(k+m-l) !} \delta_{k+m-l, 0} \\
& =\frac{(-1)^{l-m} n ! l !}{(l-m) !(n-l+m) !} .
\end{aligned}
$$

The result on the right-hand side with the part written in the general form

$$
\frac{1}{k !(n-k) !} \neq 0 \Rightarrow 0 \leq k \leq n \text {. }
$$

can be only different from zero for integer $k$ such as written if it is non-negative and not greater than $n$. If such expressions are within a summation this may restrict the bounds for the summation.

The more detailed derivation of (5.4) using (B1) of Appendix B and then the disentanglement relation (B3)) is the following

$$
\begin{aligned}
E_{\mu}(k+1 ; z) & =(1-z)^{\mu(k+1)+1} \frac{\partial^{\mu}}{\partial z^{\mu}} z^{\mu} \frac{E_{\mu}(k ; z)}{(1-z)^{\mu k+1}} \\
& =\left(\frac{\partial}{\partial z}+\frac{\mu(k+1)+1}{1-z}\right)^{\mu} z^{\mu} \frac{(1-z)^{\mu(k+1)+1}}{(1-z)^{\mu k+1}} E_{\mu}(k ; z) \\
& =\left(\frac{\partial}{\partial z}+\frac{\mu(k+1)+1}{1-z}\right)^{\mu} z^{\mu}(1-z)^{\mu} E_{\mu}(k ; z) \\
& =\sum_{i=0}^{\mu} \frac{\mu !(\mu(k+1)+1) !}{i !(\mu-i) !(\mu k+1+i) !} \frac{\partial^{i}}{\partial z^{i}} \frac{z^{\mu}(1-z)^{\mu}}{(1-z)^{\mu-i}} E_{\mu}(k ; z) \\
& =\sum_{l=0}^{\mu} \frac{\mu !(\mu(k+1)+1) !}{i !(\mu-i) !(\mu k+1+i) !}\left(\frac{\partial^{i}}{\partial z^{i}} z^{\mu}(1-z)^{i}\right) E_{\mu}(k ; z) \\
& =\sum_{i=0}^{\mu} \frac{\mu !(\mu(k+1)+1) !}{i !(\mu-i) !(\mu k+1+i) !} \sum_{m=0}^{\mu k}\left(\frac{\partial^{i}}{\partial z^{i}} z^{\mu+m}(1-z)^{i}\right) E_{\mu}(k ; m) .
\end{aligned}
$$

A full representation of the right-hand side in powers of $z$ alone is obtained by Taylor series expansion of $(1-z)^{i}$ but this makes the right-hand side to a triple sum. 
Using (A1) with corresponding parameter substitutions we find from (5.9)

$$
\begin{aligned}
& E_{\mu}(k+1, l) \\
& =\frac{1}{l !}\left\{\frac{\partial^{l}}{\partial z^{l}} \sum_{i=0}^{\mu} \frac{\mu !(\mu(k+1)+1) !}{i !(\mu-i) !(\mu k+1+i) !} \sum_{m=0}^{\mu k}\left(\frac{\partial^{i}}{\partial z^{i}} z^{\mu+m}(1-z)^{i}\right) E_{\mu}(k, m)\right\}_{z=0} \\
& =\frac{1}{l !} \sum_{i=0}^{\mu} \frac{\mu !(\mu(k+1)+1) !}{i !(\mu-i) !(\mu k+1+i) !} \sum_{m=0}^{\mu k} \frac{(-1)^{i-\mu-m+l} i !(l+i) !}{(i-\mu-m+l) !(\mu+m-l) !} E_{\mu}(k, m) \\
& =\sum_{i=0}^{\mu} \frac{(-1)^{i} \mu !(\mu(k+1)+1) !(l+i) !}{(\mu-i) !(\mu k+1+i) ! l !} \sum_{m=l-\mu}^{i+l-\mu} \frac{(-1)^{l-\mu-m}}{(i-\mu-m+l) !(\mu+m-l) !} E_{\mu}(k, m) \\
& =\sum_{i=0}^{\mu} \frac{\mu !}{i !(\mu-i) !} \frac{(\mu(k+1)+1) !(l+i) !}{(\mu k+1+i) !} \frac{\left(\sum_{j=0}^{i} \frac{(-1)^{i-j} i !}{j !(i-j) !} E_{\mu}(k, l-\mu+j)\right) .}{}
\end{aligned}
$$

By substitutions of the summation variables $j=\mu-n$ and $i=\mu-m$ this can be transformed to the form

$$
\begin{aligned}
& E_{\mu}(k+1, l) \\
& =\sum_{n=0}^{\mu} \frac{\mu !}{n !(\mu-n) !}\left(\sum_{m=0}^{n} \frac{(-1)^{n-m} n !}{m !(n-m) !} \frac{(\mu(k+1)+1) !}{(\mu(k+1)+1-m) !} \frac{(l+\mu-m) !}{l !}\right) E_{\mu}(k, l-n) .
\end{aligned}
$$

The inner sum can be expressed by a specialized Jacobi polynomial $\mathrm{P}_{n}^{(\alpha, \beta)}(u)$ for the special value $u=-1$ (or $u=+1$ by a transformation) according to

$$
E_{\mu}(k+1, l)=\mu ! \sum_{n=0}^{\mu} \frac{(l+\mu-n) !}{l !(\mu-n) !} \mathrm{P}_{n}^{(\mu(k+1)+1-n, l-\mu k-1-n)}(-1) E_{\mu}(k, l-n) .
$$

Jacobi polynomials for argument $u=\mp 1$ can be evaluated as follows

$$
\mathrm{P}_{n}^{(\alpha, \beta)}(-1)=(-1)^{n} \mathrm{P}_{n}^{(\beta, \alpha)}(1)=(-1)^{n} \frac{(n+\beta) !}{n ! \beta !} .
$$

This leads to the final expression

$$
\begin{aligned}
E_{\mu}(k+1, l) & =\mu ! \sum_{n=0}^{\mu} \frac{(l+\mu-n) !}{l !(\mu-n) !} \frac{(-1)^{n}(l-\mu k-1) !}{n !(l-\mu k-1-n) !} E_{\mu}(k, l-n) \\
& =\mu ! \sum_{n=0}^{\mu} \frac{(l+\mu-n) !}{l !(\mu-n) !} \frac{(n+\mu k-l) !}{n !(\mu k-l) !} E_{\mu}(k, l-n) .
\end{aligned}
$$

where we used in second line a transformation from factorials for negative numbers to that for positive numbers (see, e.g. [12]; is also easily derivable).

Although we derived a reliable and checked result for $E_{\mu}(k+1, l)$ in relation to $E_{\mu}(k, m)$ it was obtained in not very easy way using a lot of transformations and it is not yet clear whether or not this is the best way. In particular, it seems to be possible to derive it with normal instead of anti-normal ordering but this is also not simple ${ }^{3}$.

${ }^{3}$ It was not a principal reason to use anti-normal ordering for the proof. We tried it also by normal ordering but came first with anti-normal ordering to the result. It is also possible that some intermediate steps in the proof can be shortened. 


\section{Appendix B}

\section{Some ordering relations for differential and multiplication operators}

We give here a few ordering relations for differential operators $\frac{\partial}{\partial z}$ and multiplication operators with powers of the functions $1-z$ which we use for proofs in this paper. First, we mention the simple commutation rules

$$
\begin{aligned}
& (1-z)^{\alpha} \frac{\partial}{\partial z}=\left(\frac{\partial}{\partial z}+\frac{\alpha}{1-z}\right)(1-z)^{\alpha} \\
& \Rightarrow(1-z)^{\alpha} \frac{\partial^{n}}{\partial z^{n}}=\left(\frac{\partial}{\partial z}+\frac{\alpha}{1-z}\right)^{n}(1-z)^{\alpha},
\end{aligned}
$$

and analogously

$$
\frac{1}{(1-z)^{\beta}} \frac{\partial^{n}}{\partial z^{n}}=\left(\frac{\partial}{\partial z}-\frac{\beta}{1-z}\right)^{n} \frac{1}{(1-z)^{\beta}},
$$

where $\alpha$ are arbitrary real (or even complex) numbers. Next we consider the disentanglement of the operators $\left(\frac{\partial}{\partial z}+\frac{\alpha}{1-z}\right)^{n}$ that means its representation in anti-normal or in normal ordering.

The anti-normal ordering (all powers of the differential operators $\frac{\partial}{\partial z}$ are in front of multiplication operators) is

$$
\begin{aligned}
\left(\frac{\partial}{\partial z}+\frac{\alpha}{1-z}\right)^{n} & =\sum_{j=0}^{n} \frac{n ! \alpha !}{j !(n-j) !(\alpha-j) !} \frac{\partial^{n-j}}{\partial z^{n-j}} \frac{1}{(1-z)^{j}} \\
& =\sum_{i=0}^{n} \frac{n ! \alpha !}{i !(n-i) !(\alpha-n+i) !} \frac{\partial^{i}}{\partial z^{i}} \frac{1}{(1-z)^{n-i}} .
\end{aligned}
$$

Let us prove this relation by complete induction. The relation is obviously true for $n=0$ and for $n=1$. We suppose that it is true for arbitrary $n$ and show that it is right then also for $n+1$

$$
\begin{aligned}
\left(\frac{\partial}{\partial z}+\frac{\alpha}{1-z}\right)^{n+1} & =\sum_{j=0}^{n} \frac{n ! \alpha !}{j !(n-j) !(\alpha-j) !} \frac{\partial^{n-j}}{\partial z^{n-j}} \frac{1}{(1-z)^{j}}\left(\frac{\partial}{\partial z}+\frac{\alpha}{1-z}\right) \\
& =\sum_{j=0}^{n} \frac{n ! \alpha !}{j !(n-j) !(\alpha-j) !} \frac{\partial^{n+1-j}}{\partial z^{n+1-j}} \frac{1}{(1-z)^{j}} \\
& +\sum_{j=0}^{n} \frac{n ! \alpha !(\alpha-j)}{j !(n-j) !(\alpha-j) !} \frac{\partial^{n-j}}{\partial z^{n-j}} \frac{1}{(1-z)^{j+1}} \quad(j \rightarrow j-1) \\
& =\sum_{j=0}^{n} \frac{n ! \alpha !((n+1-j)+j)}{j !(n+1-j) !(\alpha-j) !} \frac{\partial^{n+1-j}}{\partial z^{n+1-j}} \frac{1}{(1-z)^{j}} \\
& =\sum_{j=0}^{n} \frac{(n+1) ! \alpha !}{j !(n+1-j) !(\alpha-j) !} \frac{\partial^{n+1-j}}{\partial z^{n+1-j}} \frac{1}{(1-z)^{j}},
\end{aligned}
$$

and (B3) is proved.

The normal ordering (all powers of the differential operators $\frac{\partial}{\partial z}$ are behind 
of multiplication operators) is

$$
\begin{aligned}
\left(\frac{\partial}{\partial z}-\frac{\beta}{1-z}\right)^{n} & =\sum_{j=0}^{n} \frac{(-1)^{j} n ! \beta !}{j !(n-j) !(\beta-j) !(1-z)^{j}} \frac{1}{\partial z^{n-j}} \\
& =\sum_{i=0}^{n} \frac{(-1)^{n-i} n ! \beta !}{i !(n-i) !(\beta-n+i) !} \frac{1}{(1-z)^{n-i}} \frac{\partial^{i}}{\partial z^{i}} .
\end{aligned}
$$

The proof can be made by complete induction in analogy to the proof of (3). More general operator identities of such kind are derived in [11]. The operator identities (B3) and (B5) are applicable to arbitrary functions of $z$.

If we substitute in (B3) $\alpha \rightarrow-\beta$ then we find in anti-normal ordering

$$
\begin{aligned}
\left(\frac{\partial}{\partial z}-\frac{\beta}{1-z}\right)^{n} & =\sum_{j=0}^{n} \frac{n !(-\beta) !}{j !(n-j) !(-\beta-j) !} \frac{\partial^{n-j}}{\partial z^{n-j}} \frac{1}{(1-z)^{j}} \\
& =\sum_{j=0}^{n} \frac{(-1)^{j} n !(\beta-1+j) !}{j !(n-j) !(\beta-1) !} \frac{\partial^{n-j}}{\partial z^{n-j}} \frac{1}{(1-z)^{j}},
\end{aligned}
$$

and in analogous way from (B5) by substituting $\beta \rightarrow-\alpha$

$$
\begin{aligned}
\left(\frac{\partial}{\partial z}+\frac{\alpha}{1-z}\right)^{n} & =\sum_{j=0}^{n} \frac{(-1)^{j} n !(-\alpha) !}{j !(n-j) !(-\alpha-j) !(1-z)^{j}} \frac{1}{\partial z^{n-j}} \\
& =\sum_{j=0}^{n} \frac{n !(\alpha-1+j) !}{j !(n-j) !(\alpha-1) !(1-z)^{j}} \frac{1}{\partial z^{n-j}} .
\end{aligned}
$$

We have here applied a relation

$$
\frac{(-a) !}{(-a-k) !}=(-1)^{k} \frac{(k+a-1) !}{(a-1) !}, \quad(k=0, \pm 1, \pm 2, \cdots),
$$

between factorials of positive and negative numbers with integers $k$ (e.g., Gradshteyn and Ryzhik, [12], chap. 8.334). 


\section{Appendix C}

\section{Ordering relations involving the Stirling numbers}

We collect in this Appendix some ordering relations for operators of differentiation and multiplication which involve the Stirling numbers of first kind $s(k, l)$ and of second kind $S(k, l)$. The Stirling numbers are discussed in most representations of combinatorics (e.g., in cited [1] [3] and well represented by van Lint and Wilson in [9]). We begin with Stirling numbers of second kind.

The basic definition of Stirling numbers of second kind $S(k, l)$ is by the relation between $z^{k}$ and $\frac{z !}{(z-l) !}$ as follows ${ }^{4}$

$$
z^{k}=\sum_{l=0}^{k} S(k, l) \frac{z !}{(z-l) !}=\sum_{l=0}^{k}(-1)^{k-l} S(k+1, l+1) \frac{(z+l) !}{z !} .
$$

For $(z+1)^{n}$ one obtains one obtains the following two simple and often useful forms

$$
(z+1)^{k}=\sum_{l=0}^{k} S(k+1, l+1) \frac{z !}{(z-l) !}=\sum_{l=0}^{k}(-1)^{k-l} S(k, l) \frac{(z+l) !}{z !} .
$$

One of the means for proofs by complete induction of formulae is usually the recurrence relation

$$
S(k+1, l+1)=S(k, l)+(l+1) S(k, l+1) .
$$

The explicit expression for $S(k, l)$ is

$$
S(k, l)=\sum_{j=0}^{l} \frac{(-1)^{j}}{j !(l-j) !}(l-j)^{k}, S(0,0)=1,(l \leq k),
$$

where $S(0,0)$ is indeterminate by this formula and, therefore, is additionally given.

The transition from $\left(z \frac{\partial}{\partial z}\right)^{k}$ to normal ordering (all powers of $z$ are in front of all powers of $\frac{\partial}{\partial z}$ ) and to anti-normal ordering (all powers of $z$ are behind of all powers of $\frac{\partial}{\partial z}$ ) is by the relations

$$
\left(z \frac{\partial}{\partial z}\right)^{k}=\sum_{l=0}^{k} S(k, l) z^{l} \frac{\partial^{l}}{\partial z^{l}}=\sum_{l=0}^{k}(-1)^{k-l} S(k+1, l+1) \frac{\partial^{l}}{\partial z^{l}} z^{l},
$$

and the analogous transition from $\left(\frac{\partial}{\partial z} z\right)^{k}$ possesses the form

$$
\begin{aligned}
& { }^{4} \text { The use of the Pochhammer symbol }(z)_{l} \equiv \frac{z !}{(z-l) !} \text { is not unique in literature and sometimes with } \\
& \text { this symbol the expression } \frac{(z-1+l) !}{(z-1) !} \text { is denoted. Often these two possibilities are distinguished by } \\
& \text { the notations }(z)_{l} \equiv z^{!} \equiv \frac{z !}{(z-l) !} \text { and }(z)^{(l)} \equiv z^{i} \equiv \frac{(z-1+l) !}{(z-1) !}
\end{aligned}
$$




$$
\left(\frac{\partial}{\partial z} z\right)^{k}=\sum_{l=0}^{k} S(k+1, l+1) z^{l} \frac{\partial^{l}}{\partial z^{l}}=\sum_{l=0}^{k}(-1)^{k-l} S(k, l) \frac{\partial^{l}}{\partial z^{l}} z^{l}
$$

For some convenience and completeness let us give in addition the inverse relations with the Stirling numbers of first kind $s(k, l)$. They satisfy the relations

$$
\sum_{l=0}^{k} S(k, l) s(l, m)=\sum_{l=0}^{k} s(k, l) S(l, m)=\delta_{k, m} .
$$

A basic definition of $s(k, l)$ is by the relation

$$
\frac{z !}{(z-k) !}=\sum_{l=0}^{k} s(k, l) z^{l}=\sum_{l=0}^{k} s(k+1, l+1)(z+1)^{l},
$$

or equivalently

$$
\frac{(z+k) !}{z !}=\sum_{l=0}^{k}(-1)^{k-l} s(k+1, l+1) z^{l}=\sum_{l=0}^{k}(-1)^{k-l} s(k, l)(z+1)^{l},
$$

The recurrence relation is

$$
s(k+1, l+1)=s(k, l)-(k+1) s(k, l+1) .
$$

Explicit expressions for the Stirling numbers of first kind are not so simple as for the second kind and are obtained by ( $\delta_{k, j}$ Kronecker symbol; note that the $j$-s in denominator are not factorials!)

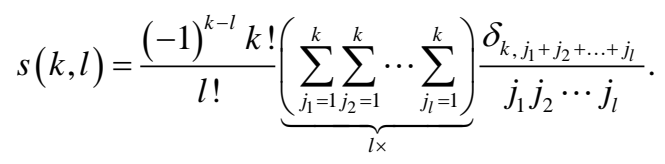

We checked these formulae by computer 5

The transition from normally ordered operators $z^{k} \frac{\partial^{k}}{\partial z^{k}}$ to $\left(z \frac{\partial}{\partial z}\right)^{l}$ or to $\left(z \frac{\partial}{\partial z}\right)^{l}$ is by the formulae

$$
z^{k} \frac{\partial^{k}}{\partial z^{k}}=\sum_{l=0}^{k} s(k, l)\left(z \frac{\partial}{\partial z}\right)^{l}=\sum_{l=0}^{k} s(k+1, l+1)\left(\frac{\partial}{\partial z} z\right)^{l},
$$

and the same from anti-normally ordered operators $\frac{\partial^{k}}{\partial z^{k}} z^{k}$

$$
\frac{\partial^{k}}{\partial z^{k}} z^{k}=\sum_{l=0}^{k}(-1)^{k-l} s(k+1, l+1)\left(z \frac{\partial}{\partial z}\right)^{l}=\sum_{l=0}^{k}(-1)^{k-l} s(k, l)\left(\frac{\partial}{\partial z} z\right)^{l} .
$$

The direct transitions from $\frac{\partial^{k}}{\partial z^{k}} z^{k}$ to $z^{k} \frac{\partial^{k}}{\partial z^{k}}$ and vice versa is given by

$$
\begin{aligned}
& \frac{\partial^{k}}{\partial z^{k}} z^{k}=\sum_{j=0}^{k} \frac{k !^{2}}{j !(k-j) !^{2}} z^{k-j} \frac{\partial^{k-j}}{\partial z^{k-j}}, \\
& z^{k} \frac{\partial^{k}}{\partial z^{k}}=\sum_{j=0}^{k} \frac{(-1)^{j} k !^{2}}{j !(k-j) !^{2}} \frac{\partial^{k-j}}{\partial z^{k-j}} z^{k-j},
\end{aligned}
$$

${ }^{5}$ With increasing $l$ under fixed $k \leq 10$ the calculation time grows very rapidly. 
and may be proved by complete induction.

A lot of formulae for the Stirling numbers can be found in the article of Bressoud [3] in the NIST handbook [4].

For convenience of using the paper in self-contained way we give here tables of Stirling numbers of second kind (Table A1) and of first kind (Table A2):

The empty places in Table A1 and Table A2 are zeros.

The connection between two different basic formulae for the Stirling numbers of second and first kind, for example, (C5) and (C1) for second kind can be established by applying (C5) to the eigenfunctions $z^{n},(n=0,1,2, \cdots)$ of the operator $z \frac{\partial}{\partial z}$ to the eigenvalue $n$ according to

Table A1. Stirling numbers of second kind $S(n, k)$.

\begin{tabular}{|c|c|c|c|c|c|c|c|c|c|c|c|c|}
\hline$n$ & $k=0$ & $k=1$ & $k=2$ & $k=3$ & $k=4$ & $k=5$ & $k=6$ & $k=7$ & $k=8$ & $k=9$ & $k=10$ & \\
\hline 0 & 1 & & & & & & & & & & & \\
\hline 1 & 0 & 1 & & & & & & & & & & \\
\hline 2 & 0 & 1 & 1 & & & & & & & & & \\
\hline 3 & 0 & 1 & 3 & 1 & & & & & & & & \\
\hline 4 & 0 & 1 & 7 & 6 & 1 & & & & & & & \\
\hline 5 & 0 & 1 & 15 & 25 & 10 & 1 & & & & & & \\
\hline 6 & 0 & 1 & 31 & 90 & 65 & 15 & 1 & & & & & \\
\hline 7 & 0 & 1 & 63 & 301 & 350 & 140 & 21 & 1 & & & & \\
\hline 8 & 0 & 1 & 127 & 966 & 1701 & 1050 & 266 & 28 & 1 & & & \\
\hline 9 & 0 & 1 & 255 & 3025 & 7770 & 6951 & 2646 & 462 & 36 & 1 & & \\
\hline 10 & 0 & 1 & 511 & 9330 & 34105 & 42525 & 22827 & 5880 & 750 & 45 & 1 & \\
\hline
\end{tabular}

Table A2. Stirling numbers of first kind $s(n, k)$.

\begin{tabular}{|c|c|c|c|c|c|c|c|c|c|c|c|c|}
\hline$n$ & $k=0$ & $k=1$ & $k=2$ & $k=3$ & $k=4$ & $k=5$ & $k=6$ & $k=7$ & $k=8$ & $k=9$ & $k=10$ & $\ldots$ \\
\hline 0 & 1 & & & & & & & & & & & \\
\hline 1 & 0 & 1 & & & & & & & & & & \\
\hline 2 & 0 & -1 & 1 & & & & & & & & & \\
\hline 3 & 0 & 2 & -3 & 1 & & & & & & & & \\
\hline 4 & 0 & -6 & 11 & -6 & 1 & & & & & & & \\
\hline 5 & 0 & 24 & -50 & 35 & -10 & 1 & & & & & & \\
\hline 6 & 0 & -120 & 274 & -225 & 85 & -15 & 1 & & & & & \\
\hline 7 & 0 & 720 & -1764 & 1624 & -735 & 175 & -21 & 1 & & & & \\
\hline 8 & 0 & -5040 & 13068 & -13132 & 6769 & -1960 & 322 & -28 & 1 & & & \\
\hline 9 & 0 & 40320 & -109584 & 118124 & -67284 & 22449 & -4536 & 546 & -36 & 1 & & \\
\hline 10 & 0 & -362880 & 1026576 & -1172700 & 723680 & -269325 & 63273 & -9450 & 870 & -45 & 1 & \\
\hline
\end{tabular}




$$
\left(z \frac{\partial}{\partial z}\right) z^{n}=n z^{n}
$$

with the consequence

$$
\left(n^{k}\right) z^{n}=\left(z \frac{\partial}{\partial z}\right)^{k} z^{n}=\left(\sum_{l=0}^{k} S(k, l) z^{l} \frac{\partial^{l}}{\partial z^{l}}\right) z^{n}=\left(\sum_{l=0}^{k} S(k, l) \frac{n !}{(n-l) !}\right) z^{n} .
$$

This means the equality (C5) for all discrete eigenfunctions $z^{n}$ of the operator $z \frac{\partial}{\partial z}$ to eigenvalues $n$. This shows the equivalence of (C1) and (C5) which both can be used for the basic introduction of the Stirling numbers of second kind. Analogous consideration can be made for the Stirling of first kind and for all inversions. 